\title{
MODIFICATIONS TO THE UNIVERSITY OF MICHIGAN 83-INCH CYCLOTRON TO IMPROVE BEAM QUALITY
}

\author{
W. C. Parkinson, W. S. Gray, J. F. Petersen and R. H. Day \\ Cyclotron Laboratory, Department of Physics
}

The University of Michigan, Ann Arbor, Michigan 48105

\begin{abstract}
Recent studies of the internal and extracted beams at the higher energies (up to $40-\mathrm{MeV}$ deuterons and $80-\mathrm{MeV} \alpha$-particles) led us to convert the dee system of the $83-$ inch cyclotron from two $150^{\circ}$ dees to one $180^{\circ}$ dee. The primary purpose was to remove from the deflector channel the rf dee voltage, which because of its phase opposes the dc deflector voltage and in addition introduces an energy spread in the extracted beam. The one-dee system offers further advantages. The equivalent first harmonic due to a gap-crossing driving force which is a function of the dee geometry and dee voltage balance in the two-dee system, is essentially eliminated, and control of the central orbits, in particular the selection of phase width, is facilitated. The measured values of the beam quality and energy spread are in good agreement with calculations. These results, together with the diagnostic instrumentation used in obtaining them, are described.
\end{abstract}

\section{INTRODUCT ION}

The University of Michigan 83-inch isochronous cyclotron was designed ${ }^{1}$ to accelerate protons to $35 \mathrm{MeV}$, deuterons to $40 \mathrm{MeV}$, and $\alpha$-particles to $80 \mathrm{MeV}$. When the cyclotron and its ancillary equipment became operational in 1964, the first interest of the users called for relatively low energy deuterons, $\alpha$-particles and ${ }^{3} \mathrm{He}$ ions, and these beams were obtained, albeit with moderate quality, without extensive studies of internal orbits. Soon, however, interest developed in spectroscopic problems that required higher particle energies and intensities and better resolution. Various attempts to obtain the higher energies were made by "emperical twiddling" by the research staff for their particular experiments. Over the past several years this has resulted in inefficient operation and not infrequently damage to components of the cyclotron. While some beams were adequate, others were not. For example the cyclotron produced a $45-\mathrm{MeV}$ a-beam essentially continuously over a three-week period with a beam current in the scattering chamber of 100 na per $E / \Delta E=8 \times 10^{3}$ (for

*Work supported in part by the U. S. Atomic Energy Commission 
most experiments, using dispersion cancellation, typical currents on the target were from 300 to $600 \mathrm{na}$ ). On the other hand, beams of $58-\mathrm{MeV}$ a-particles (and $30-\mathrm{MeV}$ deuterons) were unreliable, the quality and intensity being dependent on the tuning skill of the particular researcher.

This unsatisfactory condition of higher energy operation motivated a systematic study of the internal orbits and the extraction problem. As a result, beginning in mid-April the cyclotron was converted from a two-dee to a one-dee system. The present paper gives a brief description of the methods and conclusions of the study. Preliminary measurements made with the new one-dee system are also reported.

\section{DIAGNOSTIC INSTRUMENTATION}

Integral and differential current measurements are made with a center-line probe which moves in radius at a fixed azimuth corresponding very nearly to the center of a hill. The head of the probe used to measure the total circulating current is approximately $1-1 / 2$ inches in radial length, $3 / 8$ inches wide, and 2 inches high, filling the vertical aperture of the dee. The $\Delta R$ probe used to measure the differential current density as a function of radius is a tungsten wire, normally $0.032^{\prime \prime}$ in diameter and $2^{\prime \prime}$ long (in the $z$-direction), carried by but insulated from the probe head. The wire can be extended from the probe head toward smaller radi $i$ by as much as one inch, or it can be retracted into it. The $\Delta R$ measurements are used to determine the amplitudes of both the coherent and incoherent oscillations. At various times a second similar probe, located $180^{\circ}$ in azimuth from the center-line probe, has been used for shadow measurements as well as for $R-\Delta R$ measurements. A 3-finger probe was used in the past for measurements of the current distribution in the Z-direction, but the burn pattern technique ${ }^{2}$ gives more information and has been used to determine the vertical motion of the beam at all radii.

The phase and phase width of the circulating beam are measured with a gamma-ray time-of-flight system which measures the time interval between a fixed reference point of the $r f$ cycle and the arrival of $\gamma$-rays emitted when the beam strikes the center-line probe. This system is the principal tool for adjusting the magnetic field for isochronism. The time resolution is better than $0.8 \mathrm{~ns}$, and phase widths of $5^{\circ}$ full width at the base have been observed. Zero phase is determined by slowly sweeping the magnetic field and observing, at a fixed probe radius, the upper and lower beam cutoff points, corresponding to a $180^{\circ}$ phase shift. To determine that there are no large phase shifts at smaller radii, the magnetic field is set first at one and then the other cutoff point, and the probe is moved to a slightly smaller radius. The reappearance of beam at the smaller radius, together with a measured phase difference of $180^{\circ}$, assures that zero phase is midway between the cutoff points. The phase probe is useful not only for determining the phase and phase width of the circulating beam as a function of radius, but also for determining whether the beam blows up vertically, when it strikes the septum, 


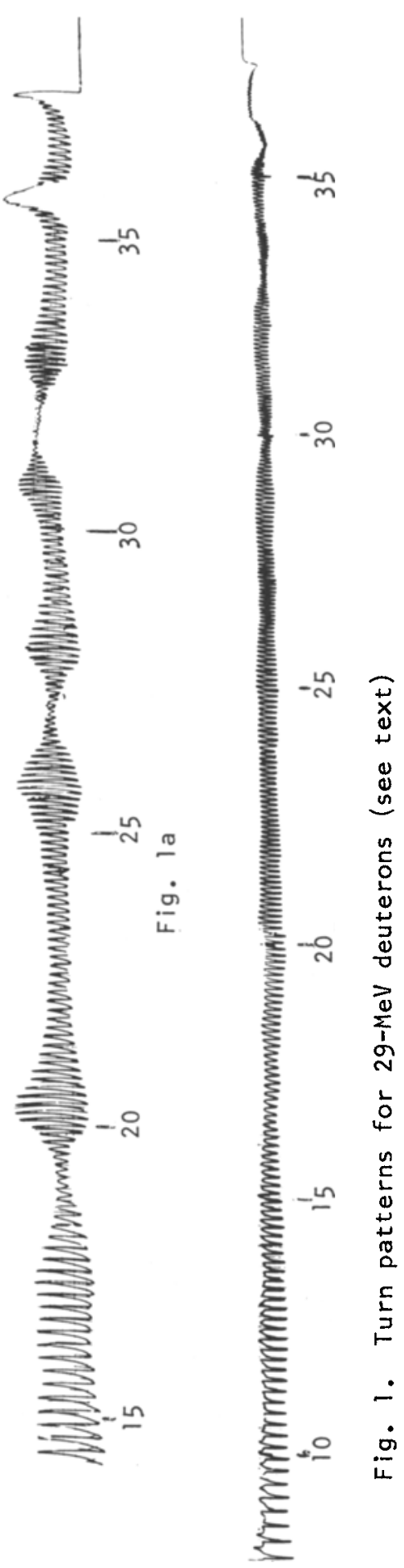

and how it moves down the extractor as the deflector voltage is raised.

The emittance $\varepsilon$ and effective source position of the extracted beam are measured using the slottedplate technique. 3 The quality of the extracted beam, by which we mean the quantity $(1 / \varepsilon) \cdot(E / \Delta E)$, is determined from the ratio of the current per unit energy interval at the foci of the first and second beam preparation magnets.

\section{DIAGNOSTIC MEASUREMENTS}

The magnetic field had been accurately measured previously. I It was remeasured during the recent shut down when Rose shims were added in the magnet gap to extend the maximum deuteron energy to 52 $\mathrm{MeV}$. Computer programs are available to predict the magnetic fields resulting from any combination of gradient coil currents. With the aid of the program, isochronous fields can be generated for any particle and energy of interest. Verification of several fields has been effected by measuring phase as a function of radius using the phase probe technique.

Median-plane electric fields have been remeasured using the electrolytic paper technique for a number of central geometries. These, together with the magnetic field data, were again used to solve numerically the equations of motion for a variety of ions in the central region for the one-dee system. The radial motion as well as the phase history was studied, and as a result of these studies, the two sets of slits, one to select phase and phase width and one to limit the amplitude of the incoherent radial oscillation, were moved to new locations. A turn pattern for 29-MeV deuterons exhibiting both coherent and incoherent oscillations is shown in Fig. la; 
the turn pattern shown in Fig. Ib, one of the best obtained, corresponds to a well centered beam with limited incoherent amplitude. Both patterns in Fig. I are for the two-dee system. A beam as well centered as in Fig. Ib has yet to be obtained with the one-dee system.

The observed sensitivity of the turn patterns to dee balance in the two-dee system lead to an investigation of the effect of the shape of the dees. Calculations indicated an effective first harmonic due to the non-radial flare in the dee gap (this is not the "gap-crossing resonance" treated by M. M. Gordon 4 but rather results from a radial component of the electric field). After the dees were altered to provide a radial flare, the sensitivity to dee balance was reduced to less than a 5 -gauss first harmonic equivalent.

A study of the Z-motion of the beam by the burn pattern technique led to the introduction of "suppressor" plates to suppress the vertical motion. The plates, one inch wide (azimuthal direction) and extending two inches in radius, were located near the center of the machine and designed to give an impulse equal and opposite to the $Z$-momentum of the beam at the instant $Z=0$. A vertical amplitude will generally result from small and unknown asymmetries in the central geometry. The use of suppressor plates provides a convenient
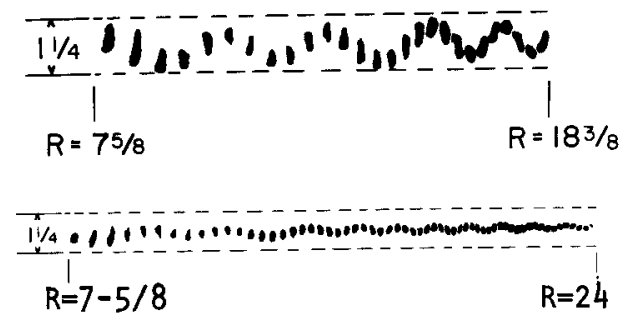

Fig. $2 a$

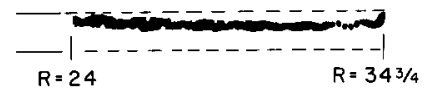

Fig. 2b

Fig. 2. Burn patterns in mylar showing the vertical distribution of the beam (see text). and effective means of reducing the vertical amplitude essentially to zero. Two such patterns are shown in Fig. 2 for a $22-\mathrm{MeV}{ }^{3} \mathrm{He}^{2+}$ beam. The pattern in $\mathrm{Fig}$. 2a shows a large vertical oscillation at small radii. The pattern in Fig. 2b was obtained with plus and minus 600 volts applied to the top and bottom suppressor plates, respectively. The pattern shows that the vertical oscillation has been suppressed. It also shows a rapid blow-up of the beam in the $+Z$ direction at $R=34^{\prime \prime}$. This sudden shift in the median surface was traced to an asymmetry that developed in gradient coil number 8 , and because the isochronous field at the higher energies requires relatively larger

currents in coil number 8 , it is believed it was one of the factors contributing to beam instability at the higher energies.

Stability limits for radial oscillations and allowable first harmonic amplitudes were recalculated with an equilibrium orbit program. The extraction of ions of various energies was reexamined with a modified version of the orbit program, and in particular the effects of the phase of the rf voltage and the radial momentum 
(amplitude and phase of the radial oscillation) on the extracted particles was studied. It was known at the time of the original design and construction of the cyclotron that for the two-dee system ions accelerated at $0^{\circ}$ rf phase (measured with respect to the center line of the dees) would experience, at the instant of extraction, an rf voltage in the deflector channel lagging by $60^{\circ}$ and in opposition to the applied dc voltage, thus requiring a higher dc vol tage to extract the ions. It was also understood 5 that, depending on the phase width of the beam, the rf would fan out the beam and increase the radial emittance, and the aperture of the external beam handing system was increased accordingly. The point that was overlooked was that the rf voltage on the deflector, unlike the dc voltage, increases the energy spread of the beam since $i t$ adds a velocity or momentum component in quadrature, the magnitude of which depends on both the phase and phase width of the beam appearing at the septum and on the effective electrical length of the deflector channel. It was expected that by removing this contribution to the energy spread, the intensity of beam in the scattering chamber for a given quality would be increased by a factor of two to five. After considering other
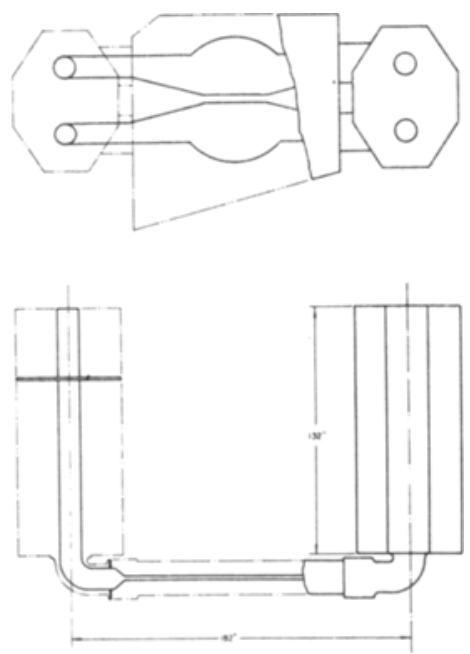

Fig. 3. The original 2-dee configuration.

obtained for 11.4-MeV deuterons. This low energy was chosen so that the number of turns to extraction could be varied easily from 175 to 370. Also, the relatively small radial gradient and resulting lower radial stability limit provided a more sensitive indication of the presence of any residual first harmonic. Preliminary measurements for the 175 turn case gave a radial emittance of $3.1 \mathrm{~mm}$ mrad for at least $50 \%$ of the beam, with an effective source width of $0.20 \mathrm{~mm}$. 
The extraction efficiency is about $85 \%$, and of the extracted beam essentially $100 \%$ appears at the image surface of the first of the two beam preparation magnets. This is to be compared with earlier measurements for 15-MeV deuterons which gave an emittance of $24 \mathrm{~mm}$ mrad for $30 \%$ of the beam with an effective source width of $0.51 \mathrm{~mm}$, an extraction efficiency of $75 \%$ with only $30 \%$ of the extracted beam appearing at the focus of the first beam preparation magnet. Normalizing the emittance measured for $11.4-\mathrm{MeV}$ deuterons to $15 \mathrm{MeV}$, the radial emittance of $2.7 \mathrm{~mm}$ mrad for $50 \%$ of the beam is to be compared with $24 \mathrm{~mm}$ mrad for $30 \%$ of the beam.

At the moment the 370 turn case has not been properly tuned. The measured emittance and source width are essentially identical to the 170 turn case, although because the orbits were less well centered, critical tuning was required to obtain a single effective source. The extraction efficiency was lower, $45-50 \%$, as was expected since the turn separation at extraction is $0.057^{11}$ compared to $0.120^{\prime \prime}$ (the septum is a carbon sheet $0.020^{\prime \prime}$ thick). Also, less than $50 \%$ of the extracted beam appeared at the focus of the first beam preparation magnet. Because the turn separation for $52-\mathrm{MeV}$ deuterons will be even less, it is planned to install a short ( $5^{\prime \prime}$ long), thin $\left(0.010^{\prime \prime}\right)$ pre-deflector $35^{\circ}$ upstream of the deflector to enhance the turn separation at the septum.

Preliminary results for $44-\mathrm{MeV}{ }^{3} \mathrm{He}$ give $4 \mathrm{~mm}$ mrad emittance for $50 \%$ of the beam and $0.3 \mathrm{~mm}$ source width, and an extraction efficiency of $50 \%$.

\section{REFERENCES}

1. W. C. Parkinson and R. S. Tickle, Nucl. Inst. \& Meth. 18, 19, 93 (1962).

2. D. J. Clark, IEEE Trans., NS-13, 15 (1966).

3. H. A. Grunder et al., Proc. Intern. Conf. Sector-focused Cyclotrons, CERN $\overline{63}-19,59$ (1963).

4. M. M. Gordon, Nuch. Instr. \& Meth. 18, 19, 268 (1962).

5. See for example the discussion on $p .110$ of reference 1 . 


\section{DISCUSSION}

RESMINI: Could you comment on the energy spread of your beams and the maximum current you expect?

PARKINSON: The energy spread of the extracted beam? I don't really know what it is now. You remember our beam preparation system--it consists of two magnets following the extractor. We have slits at th image surface of the first magnet; those slits then form the object of the second magnet which is imaged into the centre of the scatterin chamber. So the crucial ratio is how much beam out of the extracted beam that you actually get into the scattering chamber. You shouldn' hold me to these numbers 1 am going to quote. In fact, in talking with Jay Petersen this morning we have a different recollection, and unfortunately I didn't write them down. Of the $100 \%$ of the beam extracted--this is at $11.4 \mathrm{MeV}--100 \%$ of that gets to the first slits. of that it varies from $1 / 7$ to $1 / 20$ in the scattering chamber. But this is not for the full resolution. This is not $E / \Delta E$ of $8 \times 10^{3}$ but was something four times that. Certainly I don't think it is comparable to what Blosser is doing; on the other hand, we may eventually get close to that.

WILLAX: Have you made any measurements of the vertical density distribution of the external beam when you have a coherent oscillation amplitude vertically?

PARKINSON: No, we haven't, we have yet to do that. We do have a fluorescent plate which we put up after the slotted radial emittance measuring plate, and with a two-dee system as you tune the machine, i.e. tune the RF voltage or change dee voltage slightly, you can see the intensity move up and down, so there was some focusing. But we don't have a measure of it.

MILLER: Could you clarify for me once again why you would expect, going from two $180 \mathrm{deg}$ dees to one $180 \mathrm{deg}$ dee, to decrease the first harmonic driving force?

PARKINSON: I guess I didn't get this point across. With the two-dee system the dees were flared at roughly $150 \mathrm{deg}$ angle but not along a radius. So one of the things that we did was to change that flare angle, still with the two-dee system, to something in which the angle was essentially the same, but now the radial flare was symmetrical and it was a radius vector from the centre of the machine. This is what reduced that equivalent first harmonic.

SCHUTTE: You mentioned phase probe. Could you comment on that? Is it an interceptive one or non-interceptive one? What is the sensitiv ity? Capacitive or inductive?

PARKINSON: The phase probe is essentially a time-of-flight device. The timing is started by a pick-off point on the RF signal. The beam 
is allowed to hit the radial probe, which can be moved to any radius, and you have $\gamma$-rays produced on the probe. Depending on the phase of the beam with respect to the RF, you have time lag; you also have a flight time to the detector, but you can easily take that into account. It is a simple time-of-flight scheme for measuring the phase of the beam with respect to a fixed reference point on the RF. It is essentially the same system that we use for neutron time-of-flight except that we have a single small detector. Time resolution is of the order $8 / 10 \mathrm{nsec}$, not as good as some other people are doing.

It is very useful, we have used it to set 0 deg phase, for example. If we set the current probe at a given radius, let's say 25 in., then vary the magnetic field slowly, completely through resonance till we lose the beam both sides, then you are sweeping the beam through 180 deg provided there are no major excursions inside. We test this by now running the probe back in just an inch or two, and if the beam comes back at these cut-off points and if the time interval is $180 \mathrm{deg}$, then we think we know what 0 phase is. It also tells us when we get vertical blow-up of beam. We saw this at $34 \mathrm{in}$. because the dees were enough off line, $15 \mathrm{deg}$, so that we could actually measure the time difference between the spike of $\gamma$-rays corresponding to the beam hitting the dee lip as opposed to the centre-line. And we see it on the deflector, too.

RESMINI: Maybe you did quote it, but what is the dee voltage stability you have now?

PARKINSON: I don't honestly know but $I$ think it is about a part in $10^{4}$. There is a very nice way of measuring it, of course, and that is to measure the beam itself.

RESMINI: Well, I was just wondering the dee voltage at which you took those single-turn pattern pictures.

PARKINSON: Well, let me tell you. If you set the wire probe on the side of one of those turns and just let the machine sit there, then the question is, does it stay right there. Now you can see a change in current for a few mils change in radial position which you can then relate to a change in momentum and a change in dee voltage. It is a good question because I think our stability is something on the part of $10^{4}$ determined that way. Our regulator system for the coarse regulation consists of an inductive loop which is a very simple device; but instead of having a servo system which has a continuous drive, we have a stepping motor, and of course we can only go in steps. So now you can sit on the side of one of these turns and you can watch the stepping motor doing its job, and when it does you see the current change. Of course, this is not good, and we will eventually have to go to a continuous drive servo system. 